\title{
Case Study: Differential Effects of Melody and Rhythm in Melodic Intonation Therapy
}

\author{
Taiwo Whetstone, Sojung Kim, Colleen Reynolds, Emily Deeves \\ Department of Communication Sciences and Disorders, West Chester University, West Chester, United States
}

The purpose of the current observational study was to explore differential effects of melodyonly condition and rhythmic left-hand tapping-only condition in Melodic Intonation Therapy, when accompanied with formulaic words or phrase and slow rate. The participant included a 77-year-old, monolingual, non-Hispanic, Caucasian male with severe Broca's aphasia enrolled at a university clinic. The independent variable was the individual component of MIT, with two conditions: (1) melody only and (2) rhythm and left-hand tapping only. The dependent variable was percentage accuracy of the participant's ability to repeat a list of targets (i.e., pre-selected formulaic words/phrases). An alternating treatment design over two treatment phases was used in the present study. The percentage of nonoverlapping data was calculated by dividing the number of treatment sessions exceeding the highest data point during baseline sessions by the total number of treatment sessions. Results indicated that melody-only condition was a fairly effective treatment while rhythm and left-hand tappingonly condition was an unreliable treatment. The participant had difficulty following rhythm and left-hand tapping possibly due to a rhythm-processing deficit. Motivation and mood effect of singing in unison might positively influence the accuracy of repetitions in the participant. Limitations of the study were discussed.

Keywords: Melody, Rhythm, Formulaic speech, Melodic Intonation Therapy (MIT), Broca's aphasia

\section{INTRODUCTION}

Evidence has been well accumulated for more than two centuries that a patient with non-fluent, or Broca's, aphasia secondary to a left hemisphere stroke is able to sing songs and words (e.g., Marina, Pasqualetti, \& Carlomagno, 2007; Mills, 1904, Gerstmann, 1964, Stahl, Kotz, Henseler, Turner, \& Geyer, 2011). The rationale behind this evidence relies on neuroplasticity that a patient's intact right hemisphere, known to play an important role in processing steady-state spectral information and slowly changing aspects of the auditory-signal (e.g., prosodic contours and sustained vowels), may stimulate the damaged left hemisphere responsible for integrating auditory information across shorter intervals of time (Naeser \& Helm-Estabrooks, 1985; Sparks \& Deck, 1994; Zatorre \& Belin, 2001; Zipse, Worek, Guarino, \& Shattuck-Hufnagel, 2014).

This clinical observation has led to a variety of singing-based therapeutic techniques such as Melodic Intonation Therapy (MIT; Albert, Sparks, \& Helm, 1973; Helm-Estabrooks, Nicholas, \& Morgan, 1989; Sparks, Helm, \& Albert, 1974; Wan, Ruber, Hohmann, \& Schlaug, 2010). Techniques of MIT include sequentially training a patient to sing

\section{(D) \\ Check for
updates (জ) \\ Received: March 16, 2018 Accepted: August 25, 2018 \\ Correspondence: \\ Sojung Kim \\ Department of Communication Sciences and Disorders, West Chester University, West Chester, PA 19383, United States Tel: +610-436-2367 \\ Fax: +610-436-3388 \\ E-mail: skim@wcupa.edu}

(C) 2018 The Korean Association of SpeechLanguage Pathologists

This is an Open Access article distributed under the terms of the Creative Commons Attribution NonCommercial License (http://creativecommons.org/ Commercial License (http://creativecommons.org/
licenses/by-nc/4.0/) which permits unrestricted noncommercial use, distribution, and reproduction in any medium, provided the original work is properly cited. 
simple and familiar words and phrases with melody attached. While singing, a therapist rhythmically taps the patient's left hand. In other words, MIT consists of three key elements: (1) melody, (2) rhythmic tapping, and (3) formulaic, or simple and familiar (e.g., "How are you?") words or phrases.

Although the efficacy of MIT on the improvement of verbalization for patients with Broca's aphasia has been substantially documented (e.g., Conklyn, Novak, Boissy, Bethoux, \& Chemali, 2012; Hurkmans et al., 2012; Schlaug, Marchina, \& Norton, 2009), there is an ongoing debate as to which element of the three is the driving force of the efficacy of MIT or singing-based therapeutic approaches (e.g., Boucher, Garcia, Fleurant, \& Paradis, 2001; Zipse et al., 2014). The original authors of MIT considered the melody as the key component for stimulating the use of intact right hemisphere (Albert et al., 1973) or supporting the right hemisphere to activate residual left-hemisphere's function (Sparks et al., 1974). Two case observations of MIT or modified MIT attempted to highlight the crucial role of melody controlling for rhythmic left-hand tapping. Hough (2010) reported that one participant with chronic Broca's aphasia, who exhibited little progress with MIT due to difficulty with the tapping element of MIT, significantly increased production of short phrases using MIT without rhythmic tapping. Wilson, Parsons, and Reutens (2006) concluded that melody might account for the effect of MIT in a neurologically stable amateur male musician with severe expressive aphasia using a controlled pre- and post-treatment design. Consequently, these results appear to be somewhat confounded with the study participants' unique characteristics (i.e., difficulty in the tapping element and musical training prior to stroke).

The most common finding in both cross-sectional speech facilitation studies and longitudinal treatment studies that attempt to test melody-only, rhythm-only, and combined conditions is that rhythmic condition may be an essential component of the efficacy of MIT (Boucher et al., 2001; Stahl, Henseler, Turner, Geyer, \& Kotz, 2013; Stahl et al., 2011). Boucher et al. (2001) suggested that the experimental condition emphasizing rhythm resulted in significant improvements in two participants' repetition of target utterances, compared to no or little gain following the treatment condition emphasizing melody. Stahl et al. (2011) concluded that the facilitating effect of MIT has its root in rhythm rather than melody. A relatively large number of participants $(n=17)$ and rigorous study design (e.g., controlling for lip-reading, memory, and motor automaticity) allow the finding to be more credible than other case studies. Findings from a longitudinal experiment including 15 participants also confirmed that melody may not benefit speech recovery over and above rhythmic speech (Stahl et al., 2013).

In terms of formulaic speech, it is presumed that the production of common and familiar utterances (e.g., "Happy birthday to you") is automatized at the motor level. Preserved automaticity in formulaic phrases for MIT revealed that memory and motor automaticity mediate speech production in patients with Broca's aphasia (Stahl et al., 2011). In other words, individuals with non-fluent aphasia can repeat or produce formulaic utterances easier than propositional (i.e., nonformulaic, creative) speech, utilizing long-term memory and automaticity. Van Lancker Sidtis (2004) and Stahl et al. (2013) proposed that propositional and formulaic speech rely on different neural pathways. The researchers suggested that a patient with non-fluent aphasia should improve propositional speech, reactivating left hemisphere, and formulaic speech, stimulating intact right hemisphere, respectively.

In summary, researchers attempted to better understand the contribution of individual MIT components to its therapeutic effect. It is suggested that production of formulaic utterances for patients with non-fluent aphasia should be trained rhythmically. Until now the effect of melody on speech production in patients with Broca's aphasia has been somewhat ambiguous, possibly due to lack of the rigor of prior studies. The current case study was designed to demonstrate differential effects of melody-only and rhythm and lefthand tapping-only conditions on accuracy of repetitions in a patient with severe Broca's aphasia. Formulaic utterances were used in both conditions given the participant's severe deficit in propositional speech. Considering the nature of case observational study, it is not possible to eliminate extraneous variables or biases. However, the authors hope to demonstrate the functional relationship between individual MIT elements and its therapeutic effect, carefully controlling for the melodyonly and rhythm and left-hand tapping-only conditions.

\section{METHODS}

\section{Participant}

The participant of the present study was a 77-year-old, monolingual, non-Hispanic, Caucasian male with severe Broca's aphasia and apraxia of speech enrolled at a university clinic. The participant was a retired civil engineer who suffered from a left hemisphere cerebrovascular accident (CVA) six years 
prior to the intervention study, characterized with severe expressive aphasia, mild to moderate receptive aphasia, and several oral and verbal apraxia. Immediately after the onset of CVA, the participant had been treated with acute and rehabilitation therapeutic services for one year. After then, he had continued to receive speech and language services at the university clinic until the present intervention study was initiated. Previous intervention services in expressive language and speech focused on the following: (1) improving oral communication skills by improving repetition and object naming, (2) extending length of utterances, (3) improving word retrieval skills, and (4) increasing functional communication skills via use of a speech-generating AAC device. The participant's wife and previous clinicians reported that a plateau in his verbal communication ability had existed for more than one year prior to the intervention study.

The first author, a second-year graduate clinician at the time of data collection, tested the participant one week prior to the study under direct supervision of the third author, who is a certified and licensed SLP with extensive experience in the diagnosis and treatment of neurogenic disorders in adults, using Boston Diagnostic Aphasia Examination-Third Edition (BDAE-3; Goodglass, Kaplan, \& Barresi, 2001). The fourth author, a senior undergraduate student at the time of data collection, videotaped the two testing sessions. A summary of selected test results is presented in Table 1.

\section{Variables}

The independent variable was the individual component of MIT, with two conditions: (1) melody only and (2) rhythm and left-hand tapping only. A step-by-step description of melodyonly condition and rhythm and left-hand tapping-only condition is presented in Table 2 . The dependent variable is the percentage accuracy of the participant's ability to repeat a list of targets (i.e., pre-selected words/phrases). The length and complexity of targets depend on the severity of participant's deficit and personal functional relevance. Based on the results of BDAE-3 and intensive interview of the participant's wife and previous clinicians, we selected 250 words/phrases and their Boardmaker ${ }^{\oplus}$ pictures as the targets. Examples of targets are presented in Table 3. None of the targets were introduced to the participant prior to the study, and low baseline performance was obtained.

\section{Design}

The study was implemented during the university clinic's summer program for six weeks. An alternating treatment design over two treatment phases was used in the present study. To minimize order and sequence effects, two specific techniques were used in the study design. First, both conditions were administered every treatment session, with the order of conditions counterbalanced across sessions (e.g., melody presented first in treatment session one; rhythm and left-hand tapping presented first in treatment session two). In addition to the counterbalancing strategy, five control targets without treatment conditions were entered between two conditions in each treatment session. To control for unanticipated differences in difficulty or learnability between the target sets, another two techniques were utilized in the study design. First, two treatment phases were implemented. Implementing a second phase with a new set of targets resulted in "a withinparticipant replication of any effects" (Maas, Butalla, \& Farinella, 2012, p. 245). Also, each set of targets developed for each treatment phase were randomly selected for each session. Any differential learning might be attributed to the two conditions due to the random selection of targets.

\section{Random selection of targets}

The study included three baseline sessions, four maintenance sessions, and two follow-up sessions (i.e., nine sessions in total) when neither of the treatment conditions was presented. Among the pre-selected 250 words/phrases, 25 stimuli were randomly selected nine times to assign the nine sessions' targets. The study also included two treatment phases, and five

Table 1. Results of boston diagnostic aphasia examination-third edition

\begin{tabular}{llcc}
\hline Standard subtest & \multicolumn{1}{c}{ Rating scale } & Percentile & Severity sating \\
\hline Fluency & Phrase length & $<$ 10th & Severe \\
Repetition & Words & 10 th & Severe \\
Auditory comprehension & Basic word discrimination & 70 th & Mid-Moderate \\
& Commands & 40 th & Moderate \\
& Complex ideational material & 50 th & Moderate \\
\hline
\end{tabular}


Table 2. Descriptions of study phases

\section{Baseline, maintenance, and follow-up repetition task}

The first author is sitting across from the participant.

\section{Script}

Actions

Take a look at the picture on the card. The phrase (or 'word' if the card has a single word on it) for this picture is (say the target once).

Please take your time and repeat what you hear me say in your regular speaking voice (If necessary, this prompt is repeated or clarified.).

After 5 targets, the first author indicates that there is a brief pause by saying "Let's pause for a moment." Then the first author continues with the list. If the participant seems as if his attention is waning, the first author may prompt him with "Are you ready?" During the sessions, the first author may inform the participant that there be brief pauses in between targets.

\section{Melody only condition}

The first author is sitting across from the participant.
Carrier phase before each card

Step 1

Humming

Step 2

Unison singing

Step 3

Unison with fading

Step 4

Immediate repetition

Step 5

Response to a question

\section{Script}

Action

Are you ready to begin? You will be asked to sing very short words or phrases, The first author holds a hand upright first with me and then by yourself.

When I hold my hand up like this (hand is in upright stop position), it means that him what those actions look like. you should only listen to what I say.

When I hold my hand open like this (hand is in horizontal open position), it means that it is your turn to join in. Do you understand? OK let's begin.

Take a look at the picture on the card. The phrase (or 'word' if the card has a single word on it) for this picture is (say the target twice slowly then begin the melody task procedures)

The first author says "listen" and hums the target twice while holding up her left hand in a vertical stop position to indicate that he should be silent at this time. The first author then sings the target twice while still holding her left hand in a stop position as the client should still be listening.

The first author holds out her hand in a horizontal open position to indicate that The first author holds out hand in open it is the patient's turn to join in. The first author and participant then sing the position. target in unison four times. If the participant is unable to do this after four repetitions, the first author may choose a new target at step 1. If the participant is able to sing along with the first author, continue on to step 3 .

The first author still has her hand open to indicate that the participant should sing with her, but at the halfway point she fades out and the participant finishes the target alone. This should be done twice. If the participant fails to continue on his own, the first author may begin with a new target at step 1. If the participant succeeds, move onto step 4.

The first author holds up her hand to indicate that the participant should stop and listen. The first author sings the target while the participant is silent. The first author then gives the signal of an open hand to indicate that it is the participant's turn to sing the target. The first author is silent during this time. If the participant fails the target, the first author may begin with a new target at step 1. If the participant succeeds, move onto step 5.
The first author holds up the picture card for the target to show the participant.

The first author puts down picture card and holds up left hand in stop position.

The first author holds out hand in open position.

The first author holds up hand in stop position.

The first author holds out hand in open position when it is time for the participant to imitate.

The first author puts down her hands after the successful repetition and immediately asks the participant, "what did you say?" The first author can hum slightly to assist in the response.

Rhythm and tapping condition

The first author is sitting across from the participant.

\section{Script}

Are you ready to begin? You will be asked to say very short words or phrases, first with me and then by yourself.

You will need to use your left hand to tap the rhythm of the phrase. I will put my hand over top of your hand to assist you when it is time. When I hold my hand The up like this (left hand is in upright stop position), it means that you should only listen to what I say.

When I hold my hand open like this (left hand is in horizontal open position), it means that it is your turn to join in. Do you understand? OK, let's begin.

\section{Action}

The first author holds hand upright and open to show the participant what those actions will look like.

The first author then demonstrates how she taps with her hand over the participant's hand. 
Table 2. Continued

\begin{tabular}{|c|c|c|}
\hline Carrier phrase before each card & $\begin{array}{l}\text { Take a look at the picture on the card, the phrase (or 'word' if the card has a } \\
\text { single word on it) for this picture is (say the target twice slowly then begin the } \\
\text { rhythmic tapping task procedures). }\end{array}$ & $\begin{array}{l}\text { The first author holds up the picture } \\
\text { card for the target to show the } \\
\text { participant. }\end{array}$ \\
\hline $\begin{array}{l}\text { Step } 1 \\
\text { Hand tapping }\end{array}$ & $\begin{array}{l}\text { The first author says "listen" and with her right hand on top of the patient's left } \\
\text { hand, taps the target while holding up her left hand in a vertical stop position } \\
\text { to indicate that the participant should be silent during this time. The first } \\
\text { author speaks the target twice in the same rhythm as the tapping while the } \\
\text { participant listens. No response is required. }\end{array}$ & $\begin{array}{l}\text { The first author puts down picture card } \\
\text { and holds up left hand in stop } \\
\text { position. } \\
\text { The first author's right hand should be } \\
\text { over the participant's left hand. }\end{array}$ \\
\hline $\begin{array}{l}\text { Step } 2 \\
\text { Unison tapping and speaking }\end{array}$ & $\begin{array}{l}\text { The first author holds out her hand in a horizontal open position to indicate that } \\
\text { it is the participant's turn to join in. The first author and participant speak the } \\
\text { target in unison while they are tapping with his left hand and her right hand } \\
\text { together. This step is repeated four times. } \\
\text { If the participant is unable to do this after four repetitions, the participant may } \\
\text { discontinue this target and move on to a new target at step 1. If the } \\
\text { participant is successful, move onto step } 3 \text {. }\end{array}$ & $\begin{array}{l}\text { The first author holds out left hand in } \\
\text { open position. } \\
\text { The first author's right hand should be } \\
\text { over the participant's left hand. }\end{array}$ \\
\hline $\begin{array}{l}\text { Step } 3 \\
\text { Unison with fading }\end{array}$ & $\begin{array}{l}\text { The first author still has her hand open to indicate that the participant should } \\
\text { speak with her, but at the halfway point the first author fades out and the } \\
\text { participant finishes the target alone. } \\
\text { This should be done twice. If the participant fails, the first author may move to } \\
\text { a new target at step 1. If the participant succeeds, move on to step } 4 \text {. }\end{array}$ & $\begin{array}{l}\text { The first author holds out hand in open } \\
\text { position. }\end{array}$ \\
\hline $\begin{array}{l}\text { Step } 4 \\
\text { Immediate repetition }\end{array}$ & $\begin{array}{l}\text { The first author holds up her hand to indicate that the participant should stop } \\
\text { and listen. The first author speaks the target while the participant listens. The } \\
\text { first author then gives the signal of an open hand to indicate that it is the } \\
\text { participant's turn to speak the target. The first author is silent during this time. } \\
\text { If the participant fails, the first author may introduce a new target at step 1. If } \\
\text { the participant succeeds, move onto step } 5 \text {. } \\
\text { The first author holds out hand in open position when it is time for the } \\
\text { participant to imitate. }\end{array}$ & $\begin{array}{l}\text { The first author holds up hand in stop } \\
\text { position. } \\
\text { The first author removes her right hand } \\
\text { from the participant's left hand. }\end{array}$ \\
\hline $\begin{array}{l}\text { Step } 5 \\
\text { Response to a question }\end{array}$ & $\begin{array}{l}\text { The first author puts down her hands after the successful repetition and } \\
\text { immediately asks the participant, "what did you say?" The first author can tap } \\
\text { the rhythm lightly to assist in the response. }\end{array}$ & $\begin{array}{l}\text { The first author's hands are now on her } \\
\text { laps or resting on the table in front of } \\
\text { her, but not assisting the participant. }\end{array}$ \\
\hline \multirow[t]{2}{*}{ End of session } & Script & Action \\
\hline & "OK. That is the end of today's session. Thank you for your hard work today." & $\begin{array}{l}\text { The first author and participant leave } \\
\text { their treatment room. }\end{array}$ \\
\hline
\end{tabular}

Table 3. Examples of Target Words and Phrases

\begin{tabular}{ll}
\hline Treatment Phase 1-1 Melody & $\begin{array}{c}\text { Treatment Phase 1-1 Rhythm } \\
\text { and Tapping }\end{array}$ \\
\hline Butter & Banana \\
Pickle & Burger \\
Hang it up & Heat it up \\
Comb my hair & Hurry up \\
Eat the soup & Cannot hear \\
I can see & Walking up \\
Take a bite & Do not know \\
Get in car & Take a ride \\
Have a seat & Hurt my toe \\
Here it is & Cut it up \\
\hline
\end{tabular}

treatment sessions were implemented in each treatment phase. The pre-selected 250 words/phrases were divided into two sets equally, and each set of 125 words/phrases was used for each treatment phase. The number of syllables, difficulty of repetition, and familiarity were carefully considered when the pre-selected 250 words/phrases were divided into the two sets. Among the pre-selected 125 words/phrases of each set, 25 stimuli were randomly selected five times to assign the five treatment sessions' targets.

The study included both a performance-based criterion (i.e., $80 \%$ correct repetition of targets on two consecutive treatment sessions) and a time-based treatment termination criterion (i.e., five treatment sessions per phase). Because the participant did not reach the performance-based criterion in 
Table 4. Study design

\begin{tabular}{|c|c|}
\hline \multirow[t]{2}{*}{ Week 1} & - Testing session 1: BDAE-3 administered \\
\hline & - Testing session 2: BDAE-3 administered \\
\hline \multirow[t]{3}{*}{ Week 2} & - Baseline session 1: 25 randomly-selected control targets presented \\
\hline & - Baseline session 2: 25 randomly-selected control targets presented \\
\hline & - Baseline session 3: 25 randomly-selected control targets presented \\
\hline \multirow[t]{3}{*}{ Week 3} & - Treatment phase 1-1: Melody first \\
\hline & - Treatment phase 1-2: Rhythm and tapping first \\
\hline & • Treatment phase 1-3: Melody first \\
\hline \multirow[t]{4}{*}{ Week 4} & - Treatment phase 1-4: Rhythm and tapping first \\
\hline & - Treatment phase 1-5: Melody first \\
\hline & - Maintenance session 1-1: 25 randomly-selected control targets presented \\
\hline & - Maintenance session 1-2: 25 randomly-selected control targets presented \\
\hline \multirow[t]{4}{*}{ Week 5} & - Treatment phase 2-1: Rhythm and tapping first \\
\hline & - Treatment phase 2-2: Melody first \\
\hline & - Treatment phase 2-3: Rhythm and tapping first \\
\hline & - Treatment phase 2-4: Melody first \\
\hline \multirow[t]{3}{*}{ Week 6} & - Treatment phase 2-5: Rhythm and tapping first \\
\hline & - Maintenance session 2-1: 25 randomly-selected control targets presented \\
\hline & - Maintenance session 2-2: 25 randomly-selected control targets presented \\
\hline One month after maintenance 2 & - Follow-up session 1: 25 randomly-selected control targets presented \\
\hline Three months after maintenance 2 & - Follow-up session 2: 25 randomly-selected control targets presented \\
\hline
\end{tabular}

both treatment phases, five treatment sessions were completed in both phases.

\section{Procedure}

Treatment and baseline sessions were administered by the first author under direct supervision of the third author. The fourth author videotaped the entire sessions for subsequent data analysis and reliability/fidelity scoring. A summary of study duration and session sequence is presented in Table 4.

\section{Baseline phase}

The baseline phase consists of three sessions. The data for the baseline phase were based on the productions on a repetition task in which the first author produced each target at a normal rate when the Boardmaker ${ }^{\odot}$ picture was presented without any other cues. No feedback was provided during the baseline task.

\section{Treatment phases}

The present study included two treatment phases, and each treatment phase consisted of five sessions. During the treatment phases, the two conditions of melody only and rhythm and left-hand tapping only were compared. Both conditions emphasized slow production of the targets, not exceeding one syllable per second. The treatment task is a repetition task in which the first author produced each target when the Boardmaker $^{\odot}$ picture was presented either with melody only or with rhythm and left-hand tapping only. Both conditions were administered every treatment session, with the order of conditions counterbalanced across sessions (e.g., melody presented first in treatment session one; rhythm and left-hand tapping presented first in treatment session two). A randomly-selected 25 targets were assigned in each treatment session. Ten targets were presented under the melody-only condition, and another ten targets were presented under the rhythm and lefthand tapping-only condition. Five control targets without treatment conditions were entered between two conditions in each treatment session.

\section{Maintenance phase}

Each treatment phase was followed by a maintenance phase, which consisted of two sessions. The data for the two maintenance phases were based on productions of a repetition task in which the first author produced each target at a normal rate 
when the Boardmaker ${ }^{\odot}$ picture was presented without any other cues. No feedback was provided during the maintenance task.

\section{Follow-up sessions}

To monitor a short-term effect of differential learning, two follow-up sessions were implemented; one session was scheduled one month following maintenance phase two, and the other session was scheduled three months following maintenance phase two. The data for the two follow-up sessions were based on productions on a repetition task in which the first author produced each target at a normal rate when the Boardmaker ${ }^{\circledR}$ picture was presented without any other cues. No feedback was provided during the follow-up task.

\section{Reliability and Fidelity}

To calculate interrater reliability of scoring of the participant's accurate repetitions, the first and fourth authors observed the entire sessions (i.e., baseline, treatment, maintenance, and follow-up sessions) and identified the number of accurate repetitions the participant exhibited through a review of the video-recorded sessions. Point-by-point comparisons were made, and interrater agreement was calculated for the entire sessions resulting in 99\% reliability. Any disagreement between the two authors was resolved by consulting with the second author. To ensure that the first author implemented the intervention study as designed, a checklist was scored by three trained independent analysts, senior undergraduate students at the time of data analyses. The three independent analysts examined the entire baseline, treatment, maintenance, and follow-up sessions for fidelity (Kaderavek \& Justice, 2010) based on viewing video-recorded sessions. Fidelity aspects scored for each condition included the number of trials and the accuracy of scripts and actions. Summing the scores across the three observers indicated $100 \%$ fidelity of baseline sessions, $97 \%$ fidelity of treatment sessions, $99 \%$ fidelity of maintenance sessions, and $100 \%$ fidelity of follow-up sessions.

\section{RESULTS}

Data are plotted in Figure 1 and summarized in Table 5. In the figure, numbers represent the percent of the participant's accurate repetitions. Different phases are demarcated by vertical dotted lines. First, visual inspection of the data was conducted as suggested by Kromrey and Foster-Johnson (1996). The participant, in general, demonstrated fluctuating and variable performances of accurate repetitions across sessions. During baseline phrase, the participant consistently demonstrated difficulty repeating targets accurately, ranging from 20 to $33 \%$ accuracy of trials. During treatment phase one, the participant distinctively demonstrated a high level of accurate repetitions in session four under melody-only condition. The participant's accurate repetitions in sessions four and five under melody-only condition were significantly superior to the rest of performances during treatment phase one. Treatment

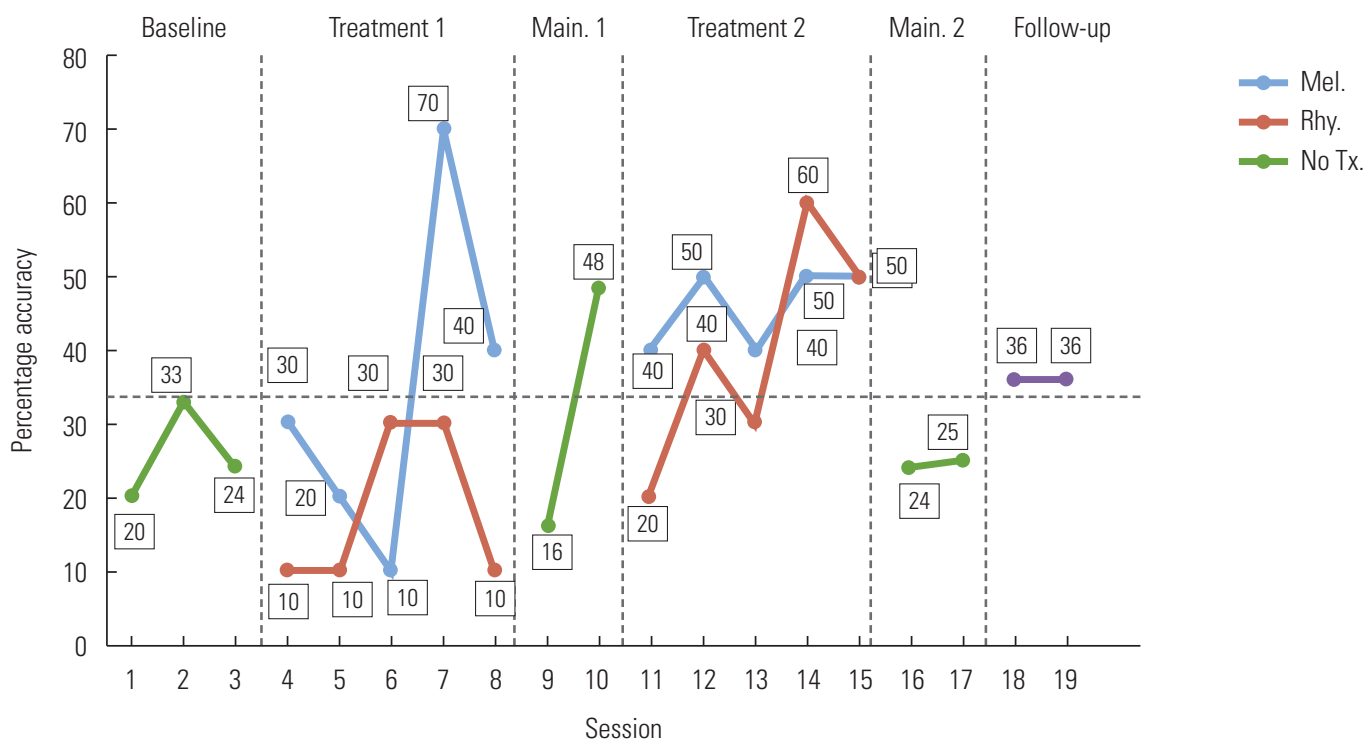

Figure 1. Percentage accuracy of the participant's correct repetitions. Mel, melody-only condition; Rhy, rhythm and tapping-only condition; No Tx, no treatment was provided. 
Table 5. Total number of trials and correct repetitions

\begin{tabular}{|c|c|c|c|c|c|}
\hline Session & Baseline 1 & Baseline 2 & Baseline 3 & Treatment1-1 & Treatment1-2 \\
\hline Correct/Total & $5 / 25$ & $4 / 12$ & $6 / 25$ & Mel. 3/10 & Mel. 2/10 \\
\hline \multirow[t]{3}{*}{$\%$} & 20 & 33.30 & 24 & 30 & 20 \\
\hline & & & & Rhy. $1 / 10$ & Rhy. $1 / 10$ \\
\hline & & & & 10 & 10 \\
\hline Session & Treatment1-3 & Treatment1-4 & Treatment1-5 & Main.1-1 & Main.1-2 \\
\hline Correct/Total & Mel. 1/10 & Mel. 7/10 & Mel. 4/10 & $4 / 25$ & $12 / 25$ \\
\hline \multirow[t]{3}{*}{$\%$} & 10 & 70 & 40 & 16 & 48 \\
\hline & Rhy. 3/10 & Rhy. 3/10 & Rhy. $1 / 10$ & & \\
\hline & 30 & 30 & 10 & & \\
\hline Session & Treatment2-1 & Treatment2-2 & Treatment2-3 & Treatment2-4 & Treatment2-5 \\
\hline Correct/Total & Mel. 4/10 & Mel. 5/10 & Mel. 4/10 & Mel. 5/10 & Mel. 5/10 \\
\hline \multirow[t]{3}{*}{$\%$} & 40 & 50 & 40 & 50 & 50 \\
\hline & Rhy. 2/10 & Rhy. 4/10 & Rhy. 3/10 & Rhy. 6/10 & Rhy. 5/10 \\
\hline & 20 & 40 & 30 & 60 & 50 \\
\hline Session & Main.2-1 & Main.2-2 & Follow-up1 & Follow-up2 & \\
\hline Correct/Total & $6 / 25$ & $6 / 24$ & $9 / 25$ & $9 / 25$ & \\
\hline$\%$ & 24 & 25 & 36 & 36 & \\
\hline
\end{tabular}

Correct, correct repetition behaviors; Total, total number of trials presented; Main, maintenance.

phase two exhibited more stable and consistent repetition patterns than treatment phase one. The participant's performances under melody-only condition were significantly better than his performances under rhythm and left-hand tapping-only condition in four sessions among five. In session five, the participant accurately repeated targets in equal numbers under both conditions.

Next, to measure the differential effect of melody-only and rhythm and left-hand tapping-only conditions, the percentage of nonoverlapping data (PND) was calculated by dividing the number of treatment sessions exceeding the highest data point during baseline sessions and dividing by the total number of treatment sessions (Parker, Hagan-Burke, \& Vannest, 2007; Manolov \& Solanas, 2009; Scruggs \& Mastropieri, 2013; Scruggs, Mastropieri, \& Casto, 1987). PND statistics higher than $90 \%$ are interpreted as highly effective intervention, $70 \%$ to $90 \%$ as fairly effective treatment, $50 \%$ to $70 \%$ as questionable effectiveness, and less than $50 \%$ as unreliable treatment. The participant's highest data point during baseline sessions is represented as a horizontal dotted line in Figure 1. PND statistic for melody-only condition is $7 / 10=70 \%$, which is fairly effective. PDN statistic for rhythm and left-hand tapping-only condition is $3 / 10=30 \%$, that is, unreliable treatment. Both follow-up sessions' data were slightly higher than the partici- pant's highest data point during baseline sessions.

\section{DISCUSSION}

The purpose of the current case study was to explore differential effects of melody-only and rhythm and left-hand tappingonly conditions on the accuracy of repetitions in a patient with severe Broca's aphasia. Formulaic utterances were used in both conditions given the participant's severe deficit in propositional speech. The study's preliminary findings have possibly added to the literature on MIT by examining a functional relationship between individual MIT elements and their effects on the accuracy of repetitions in a patient with severe Broca's aphasia. By carefully controlling for the melodyonly and rhythm and left-hand tapping-only conditions with an alternating treatment design and controls of bias (e.g., counterbalancing strategy of the two conditions, random selection of targets, and two treatment phases), these findings have possibly aimed to advance our understanding of the importance of clinical observational research for the contribution of individual MIT elements.

A major finding from the current observational study was that both visual inspection of data and PND calculation indicated an immediate effect of melody-only condition on the 
participant's accuracy of repetitions. The positive effect of rhythm and left-hand tapping-only condition was not observed. During the treatment sessions, the participant exhibited a considerable amount of impulsivity. The participant's speech was halting overall, but it was frequently observed that he rushed to complete repetition as quickly as possible. This impulsivity was accelerated during the rhythm and left-hand tapping-only condition. This condition caused the participant to move ahead of the examiner's speed, whereas this behavior did not occur during the melody-only condition. The authors' observation regarding the participant's difficulty following the tapping was consistent with rhythm-processing deficits that at least some patients with Broca's aphasia exhibit. For example, Hough (2010) reported a positive effect of MIT when the tapping element was deleted. Tapping was not presented in this study because the participant found the tapping distracting. The researcher speculated that the patient may have had impaired speech as well as impaired tapping, therefore, tapping interrupted attempts at speech production rather than facilitated them. Zipse et al. (2014) supported Hough's hypothesis that some patients with aphasia have a particular difficulty processing rhythm as opposed to pitch information. It is suggested that therapists should consider modifying MIT with the tapping omitted when (1) patients with aphasia demonstrate little success with MIT, and (2) rhythm-processing deficit is suspected.

Motivation and mood effect of singing in unison can also be considered when examining the positive effect of melodyonly condition on the accuracy of repetitions in the participant. Singing is a natural, pleasing, and stress-free way for patients with non-fluent aphasia to verbalize, and thus it possibly motivates the patients to continue to focus on verbal repetition activities (Merrett, Peretz, \& Wilson, 2014; Racette, Bard, \& Peretz, 2006). A positive relationship between music therapy and patients' motivation and mood has been substantially observed in the fields of physical rehabilitation and mental health, indicating that singing may be intrinsically motivating (Merrett et al., 2014). Considering that low mood and lack of motivation are commonly observed in individuals with aphasia (Robinson, 2003), the positive influence of singing on the participant's motivation and mood might have resulted in the improvement of accuracy of repetitions.

Limitations of the current study should be addressed. First, the results of the study were preliminary because of the selection of the participant. The participant's fatigue, distraction, and overall weakness resulted in fluctuating and variable per- formances across phases. These extraneous variables negatively influenced the study's validity given that only one participant was examined. Another limitation was that each treatment phase only included five sessions, and these were not intensive enough to examine the lasting effect of melodyonly condition during the follow-up phase. Due to the university clinic's summer schedule, the entire study including the testing, baseline, treatment, and maintenance phases had to be completed in six weeks. Although the follow-up data did not indicate the lasting effect of melody-only condition, the participant's wife and authors anecdotally reported noticeable differences in the participant's initiation of rehearsed speech at home and the clinic. For example, the participant's greeting was changed from a head nod and the phrase "okay okay" during the baseline phase, to the phrase "I am good," in later treatment sessions.

Exploring the differential effect of melody-only and rhythm and left-hand tapping-only conditions should not be viewed as a reductionist approach because clinical observations of individual conditions of MIT enrich our understanding of how MIT works for patients with Broca's aphasia. Given that some evidence for the positive effect of melody-only condition versus rhythm and left-hand tapping-only condition has been reported, attempts to find a simple and single framework explaining the effects of MIT do not appear to be worthwhile. It is possible that pre-morbid factors (e.g., musicianship), lesion factors (e.g., time since onset and location), and comorbidity factors (e.g., aphasia only and aphasia with apraxia of speech) are intertwined to determine the progress of verbalization when MIT is implemented (Merrett et al., 2014). The current case study possibly attempted to extend the literature on the functional relationship between the participant's pre-morbid, lesion, and comorbidity factors and the positive effect of individual MIT elements on the accuracy of verbal repetitions.

\section{REFERENCES}

Albert ML, Sparks RW, Helm N. Melodic intonation therapy for aphasia. Archives of Neurology. 1973;29:130-131.

Boucher V, Garcia LJ, Fleurant J, Paradis J. Variable efficacy of rhythm and tone in melody-based interventions: implications for the assumption of a right-hemisphere facilitation in non-fluent aphasia. Aphasiology. 2001;15:131-149.

Conklyn D, Novak E, Boissy A, Bethoux F, Chemali K. The effects of modified melodic intonation therapy on nonfluent aphasia: a pilot study. Journal of Speech, Language, and Hearing Research. 
2012;55:1463-1471.

Gerstmann HL. A case of aphasia. Journal of Speech and Hearing Disorder. 1964;29:89-91.

Goodglass H, Kaplan E, Barresi B. Boston Diagnostic Aphasia Examination (3rd ed.). Austin, TX: Pro-Ed. 2001.

Helm-Estabrooks N, Nicholas M, Morgan A. Melodic intonation therapy program. San Antonio, TX: Pro-Ed. 1989.

Hough MS. Melodic intonation therapy and aphasia: another variation on a theme. Aphasiology. 2010;24:775-786.

Hurkmans J, Bruijn M, Boonstra AM, Jonkers R, Bastiaanse R, Arendzen $\mathrm{H}$, et al. Music in the treatment of neurological language and speech disorders: a systematic review. Aphasiology. 2012;26:1-19.

Kaderavek JN, Justice LM. Fidelity: an essential component of evidence-based practice in speech-language pathology. American Journal of Speech-Language Pathology. 2010;19:369-379.

Kromrey JD, Foster-Johnson L. Determining the efficacy of intervention: the use of effect sizes for data analysis in single-subject research. The Journal of Experimental Education. 1996;65:73-93.

Maas E, Butalla CE, Farinella KA. Feedback frequency in treatment for childhood apraxia of speech. American Journal of SpeechLanguage Pathology. 2012;21:239-257.

Manolov R, Solanas A. Percentage of nonoverlapping corrected data. Behavior Research Methods. 2009;41:1261-1271.

Marina A, Pasqualetti P, Carlomagno S. Patterns of language improvement in adults with non-fluent aphasia after specific therapies. Aphasiology. 2007;21:164-186.

Merrett DL, Peretz I, Wilson S. Neurobiological, cognitive, and emotional mechanisms in melodic intonation therapy. Frontiers in Human Neuroscience. 2014;8:1-11.

Mills CK. Treatment of aphasia by training. The Journal of the American Medical Association. 1904;43:1940-1949.

Naeser MA, Helm-Estabrooks N. CT scan lesion localization and response to melodic intonation therapy with nonfluent aphasia cases. Cortex. 1985;21:203-223.

Parker RI, Hagan-Burke S, Vannest K. Percentage of all non-overlapping data (PAND): An alternative to PND. The Journal of Special Education. 200740:194-204.

Racette A, Bard C, Peretz I. Making non-fluent aphasics speak: sing along! Brain. 2006;129:2571-2584.
Robinson RG. Poststroke depression: prevalence, diagnosis, treatment, and disease progression. Biological Psychiatry. 2003;54:376387.

Sparks RW, Deck JW. Melodic intonation therapy. In R. Chapey (Ed.), Language intervention strategies in adult aphasia (3rd ed.) (pp. 368-379). Baltimore: Williams \& Wilkins. 1994.

Sparks RW, Helm N, Albert M. Aphasia rehabilitation resulting from melodic intonation therapy. Cortex. 1974;10:303-316.

Schlaug G, Marchina S, Norton A. Evidence for plasticity in white matter tracts of patients with chronic Broca's aphasia undergoing intense intonation-based speech therapy. Annals of the New York Academic of Sciences. 2009;1169:385-394.

Scruggs TE, Mastropieri MA. PND at 25: Past, present, and future trends in summarizing single-subject research. Remedial and Special Education. 2013;34:9-19.

Scruggs TE, Mastropieri MA, Casto G. The quantitative synthesis of single subject research methodology: Methodology and validation. Remedial and Special Education. 1987;8:24-33.

Stahl B, Henseler I, Turner R, Geyer S, Kotz SA. How to engage the right brain hemisphere in aphasic without even singing: evidence for two paths of speech recovery. Frontiers in Human Neuroscience. 2013;7:1-12.

Stahl B, Kotz SA, Henseler I, Turner R, Geyer S. Rhythm in disguise: why singing may not hold the key to recovery from aphasia. Brain. 2011;134:3083-3094.

Van Lancker Sidtis D. When novel sentences spoken or heard for the first time in the history of the universe are not enough: toward a dual-process model of language. International Journal of Language and Communication Disorders. 2004;39:1-44.

Wan CY, Ruber T, Hohmann A, Schlaug G. The therapeutic effects of singing in neurological disorders. Music Percept. 2010;27:287-295.

Wilson SJ, Parsons K, Reutens DC. Preserved singing in aphasia: a case study of the efficacy of melodic intonation therapy. Music Perfection: An Interdisciplinary Journal. 2006;24:23-36.

Zatorre RJ, Belin P. Spectral and temporal processing in human auditory cortex. Cerebral Cortex. 2001;11:946-953.

Zipse L, Worek A, Guarino AJ, Shattuck-Hufnagel S. Tapped out: do people with aphasia have rhythm processing deficits? Journal of Speech, Language, and Hearing Research. 2014;57:2234-2245. 